\section{Structure-system correlation identifies a gene regulatory Mediator submodule}

\author{
Laurent Larivière, ${ }^{1,4}$ Martin Seizl, ${ }^{1,4}$ \\ Sake van Wageningen, ${ }^{2}$ Susanne Röther, ${ }^{1}$ \\ Loes van de Pasch, ${ }^{2}$ Heidi Feldmann, ${ }^{1}$ \\ Katja Sträßer, ${ }^{1}$ Steve Hahn, ${ }^{3}$ Frank C.P. Holstege, ${ }^{2}$ \\ and Patrick Cramer ${ }^{1,5}$

\begin{abstract}
${ }^{1}$ Gene Center Munich and Center for Integrated Protein Ludwig-Maximilians-Universität München, 81377 Munich, Germany; ${ }^{2}$ Department of Physiological Chemistry, University Medical Center Utrecht, 3584 CG Utrecht, The Netherlands; ${ }^{3}$ Division of Basic Sciences, Fred Hutchinson Cancer Research Center, Seattle, Washington 98109, USA
\end{abstract} \\ Science CIPS $^{\mathrm{M}}$, Department of Chemistry and Biochemistry,
}

A combination of crystallography, biochemistry, and gene expression analysis identifies the coactivator subcomplex Med8C/18/20 as a functionally distinct submodule of the Mediator head module. Med8C forms a conserved $\alpha$-helix that tethers Med18/20 to the Mediator. Deletion of Med8C in vivo results in dissociation of Med18/20 from Mediator and in loss of transcription activity of extracts. Deletion of med8C, med18, or med20 causes similar changes in the yeast transcriptome, establishing $M e d 8 C / 18 / 20$ as a predominantly positive, genespecific submodule required for low transcription levels of nonactivated genes, including conjugation genes. The presented structure-based system perturbation is superior to gene deletion analysis of gene regulation.

Supplemental material is available at http://www.genesdev.org.

Received November 21, 2007; revised version accepted January 25, 2008.

Many different transcriptional activators and repressors regulate RNA polymerase (Pol) II transcription in eukaryotic cells. These regulatory factors generally bind to upstream DNA sites and recruit large coregulatory complexes such as the Mediator (Malik and Roeder 2000; Naar et al. 2001; Bjorklund and Gustafsson 2005; Kornberg 2005). Mediator complexes were isolated from fungi, metazoans, and a plant (Boube et al. 2002; Backstrom et al. 2007). Mediator from the yeast Saccharomyces cerevisiae ( $\mathrm{Sc}$ ) is a 1-MDa complex that comprises 25 subunits, of which 11 are essential for viability and 22 are at least partially conserved among eukaryotes. Mediator promotes initiation complex assembly through contacts with activators, Pol II, and general transcription factors.

[Keywords: Mediator of transcriptional regulation; multiprotein coactivator complex; RNA polymerase II transcription; structure-function analysis; molecular systems biology]

${ }^{4}$ These authors contributed equally to the work.

${ }^{5}$ Corresponding author.

E-MAIL cramer@Imb.uni-muenchen.de; FAX 49-89-2180-76999.

Article is online at http://www.genesdev.org/cgi/doi/10.1101/gad.465108.
Mediator subunits reside in different modules named head, middle, tail, and kinase modules (Dotson et al. 2000; Kang et al. 2001). Apparently the Mediator modules are required for the regulation of different subsets of genes. Gene deletion studies implicated the middle module in regulating HSP genes and low-iron response genes, the tail module in regulating HSP and OXPHOS genes, and the kinase module in regulating genes required during nutrient starvation (Holstege et al. 1998; Beve et al. 2005; van de Peppel et al. 2005; Singh et al. 2006).

The Mediator head module is important for initiation complex assembly, stimulates basal transcription, and is necessary for activated transcription (Ranish et al. 1999; Takagi et al. 2006). The head module contains subunits Med6, Med8, Med11, Med17, Med18, Med20, and Med22, which are conserved from yeast to human. Head subunits are essential for yeast viability, except for Med18 and Med20 (Koleske et al. 1992; Thompson et al. 1993; Lariviere et al. 2006). In vitro, Med1 8 and Med20 are required for formation of a stable initiation complex, for efficient basal transcription, and for activated transcription (Thompson et al. 1993; Lee et al. 1999; Ranish et al. 1999). In vivo, Med18 and Med20 regulate transcription of the same subset of genes and have a mainly positive function (van de Peppel et al. 2005).

Based on structural analysis, we proposed previously that the trimeric subcomplex of the C-terminal domain of Med8 (Med8C), Med18, and Med20 (the Med8C/18/20 subcomplex) forms a conserved functional submodule of the Mediator head (Lariviere et al. 2006). Here, we confirm this proposal with a combination of X-ray analysis, yeast genetics, biochemistry, and transcriptomics. Our results indicate that Mediator contains functionally distinct submodules within its previously defined modules, and show how gene regulatory submodules can be identified by a combination of structural and functional studies on the molecular level and gene expression analysis on the systems level.

Results and Discussion

Med8C/18/20 is a subcomplex of the Mediator head

Our previous analysis revealed that Sc Med8 contains an essential N-terminal domain (Med8N, residues 1-137), followed by a nonessential linker (residues 138-189) and a C-terminal region that includes a $\alpha$-helix (Med8C, residues 190-223) (Fig. 1A; Lariviere et al. 2006). We proposed that Med8C tethers the Med18/20 heterodimer to the essential part of the Mediator head (Fig. 1A). To test this, we asked whether Med8C tethers the Med18/20 heterodimer to Mediator in vivo. We isolated Mediator by tandem affinity purification (TAP) from yeast strains expressing a TAP-tagged head subunit, Med17, and identified the copurifying Mediator subunits by mass spectrometry (Fig. 1B). The same purification from a strain expressing a truncated version of Med8 that lacked Med8C $($ med8C $\Delta)$ resulted in a very similar pattern of protein bands, except that Med18 and Med20 were missing (Fig. 1B). Therefore, retention of Med18 and Med20 in the Mediator requires Med8C. The truncated Med8 variant was, however, present in the preparation, showing that Med8C is not required to retain Med8 in the Mediator. These data are consistent with interaction data 

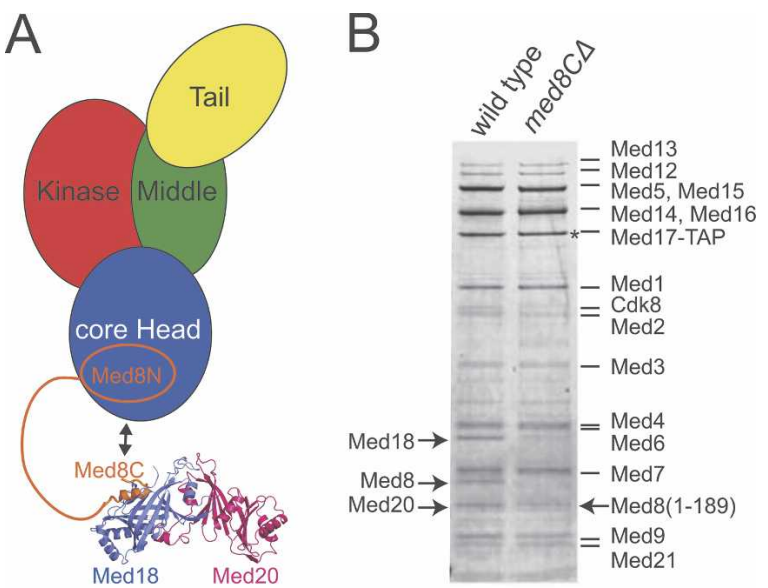

Figure 1. (A) Overview of the Mediator architecture with the four modules head, middle, tail, and kinase. The head module is separated in the core head, constituted of Med6, Med8N, Med11, Med17, Med22, and the nonessential subcomplex of Med8C, Med18, and Med20 (in orange, blue, and magenta, respectively). (B) Deletion of Med8C in vivo leads to loss of Med18/20 after Mediator purification. $\mathrm{N}$-terminally TAP-tagged Med17 was purified from wild-type yeast or from cells expressing Med $8 \Delta \mathrm{C}$. The EGTA eluate after purification was separated using $4 \%-12 \%$ discontinuous SDS-PAGE and was analyzed by mass spectrometry after Coomassie staining. The sequenced copurifying proteins are labeled. Arrows mark Med8, Med8C $\Delta$, Med18, and Med20. An asterisk indicates TAP-tagged Med17.

derived by coexpression and two-hybrid analysis (Guglielmi et al. 2004; Takagi et al. 2006) and suggest that the head contains two structural subcomplexes, the core head, consisting of all essential head subunits or subunit domains (Med6, Med8N, Med11, Med17, and Med22) and the nonessential Med8C/18/20 subcomplex (Fig. 1A).

\section{Conserved Med8C/18/20-core head interface}

Because of the low sequence homologies between Mediator subunits from different species, the architecture of the $S c$ head could be a species-specific feature. To investigate this, we solved the crystal structure of the Med8C/ 18 complex from Schizosaccharomyces pombe (Sp) (Materials and Methods). We coexpressed $S p$ Med18 with a hexahistidine-tagged $S p$ Med8C fragment corresponding to the Sc Med8C fragment used previously (Lariviere et al. 2006) from a bicistronic vector in Escherichia coli. The $S p$ Med8C fragment was sufficient for interaction with $S p$ Med18 (data not shown). The resulting stoichiometric Med8C/18 complex was crystallized and the structure solved (Materials and Methods; Supplemental Table 1). Sp Med18 adopts a fold similar to its Sc ortho$\log$ (Fig. 2A), with a root mean square deviation of $1.7 \AA$ over $173 \mathrm{C} \alpha$ atoms. $S p$ Med8C forms a $\alpha$-helix, followed by a glycine-containing turn, and binds $S p$ Med18 across its central $\beta$-barrel as observed for its $S c$ counterpart (Fig. 2A). Key contact residues in the Med8C-Med18 interface are conserved between $S c$ and $S p$ (Fig. 2B). Given the large phylogenetic distance between these two fungi, the Med8C/18 interface is apparently also conserved in Mediator complexes of higher eukaryotes. Indeed, modeling of the human Med8C-Med18 interface showed that key contacts are conserved (data not shown). Thus, the structural tethering of the Med18/20 heterodimer to the core head module through Med8C is conserved among eukaryotes.

\section{Med8C/18/20 is required for activated transcription in vitro}

To investigate whether the structural subcomplex Med8C/18/20 is also a functional subcomplex of the Mediator, we conducted in vitro transcription assays. We prepared nuclear extracts from yeast strains carrying a deletion of the gene for Med18 (med184) or lacking the part of the Med8 gene coding for Med8C (med8CA). Consistent with previous data (Thompson et al. 1993; Ranish et al. 1999|, med18D nuclear extract did not support activated transcription (Fig. 3, lane 1), apparently since Mediator in this mutant lacks both Med18 and Med20. The transcription defect could indeed be rescued by addition of recombinant Med18/20 (Fig. 3, lanes 5,6). This is consistent with the model that Med8C, which is present in the extract, tethers Med18/20 to the Mediator. Recombinant Med8C/18/20 subcomplex was far less efficient in rescue (Fig. 3, lanes 3,4), likely because endogenous Med8C fails to replace recombinant Med8C for tethering Med18/20. Consistently, a nuclear extract from the med8C $\Delta$ strain was inactive (Fig. 3, lane 1), apparently since its Mediator complex lacks Med18 and Med20. Even a large excess of Med18/20 could not rescue the defect (Fig. 3, lanes 5,6), but recombinant Med8C/18/20 could partially restore transcription (Fig. 3, lane 4). Thus, Med8C is essential for activated transcription in these assays. Since providing Med8C in trans can partially rescue the defect, Med8C apparently also interacts nonco-
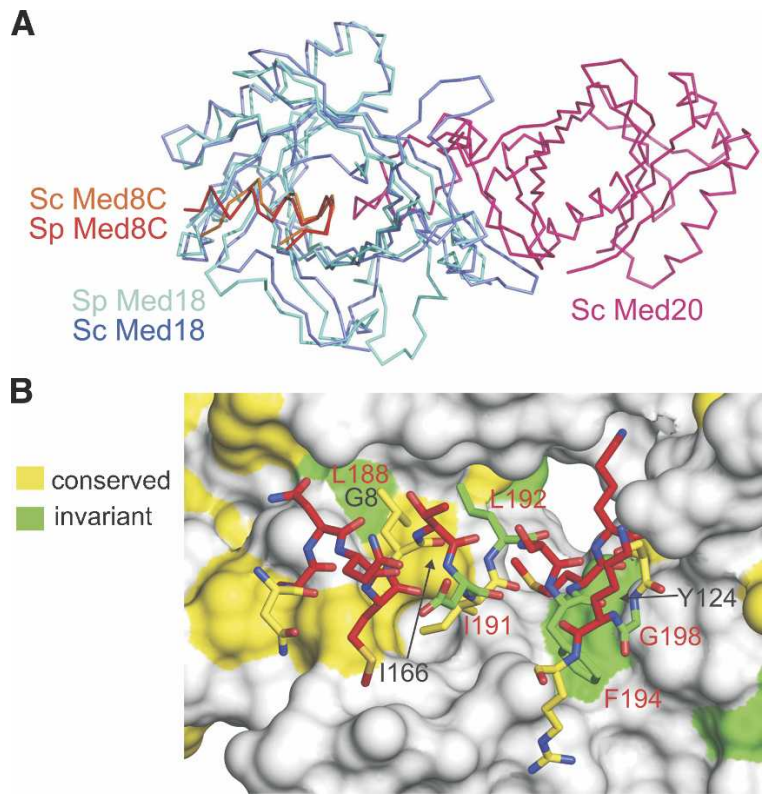

Figure 2. Structural conservation of the Med8C/18 interaction. $(A)$ Superimposition of C $\alpha$ traces of Med8C/18 from Sp (in cyan and red; this study) and from $S c$ (in blue and orange; Lariviere et al. 2006). Med20 is shown in magenta. (B) Interaction of $S p$ Med8C with $S p$ Med18. The Med8C helix is shown in red sticks, with conserved or invariant residues in contact with Med18 labeled in red. Med18 is shown as a white surface, with conserved or invariant residues in contact with Med8C labeled in black. Residues that are invariant and conserved between $S c$ and $S p$ are in green and yellow, respectively. Figures were prepared with PyMol (http://www.pymol.org). 


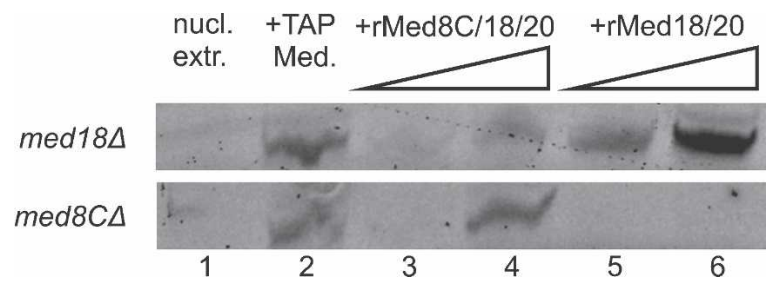

Figure 3. Med $8 \mathrm{C} / 18 / 20$ is required for activated transcription in

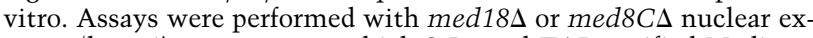
tracts (lane 1), or extract to which 0.5 pmol TAP-purified Mediator was added, providing a positive control (lane 2), or extracts to which recombinant Med8C/18/20 (10× and 100× molar excess in lanes 3,4) or recombinant Med18/20 (10x and 100x molar excess in lanes 5,6) were added.

valently with the core head, consistent with a reported two-hybrid interaction between Med8C and Med17 (Guglielmi et al. 2004). These functional data are consistent with the two-subcomplex architecture of the head (Fig. 1A), and highlight the critical functional role of Med8C in tethering the two subcomplexes together.

\section{Med8C/18/20 is a functional submodule in vivo}

To test whether the two-subcomplex architecture of the Mediator head underlies Mediator function in vivo, and to determine whether the only role of Med8C is to tether Med18/20, we carried out comparative gene expression profiling with different deletion strains of yeast. If the only role of Med8C is tethering of Med18/20 to Mediator, then the changes in gene expression observed in a Med8C deletion should be essentially the same as for deletion of either Med18 or Med20. We first used three strains of the same genetic background but specifically lacking one component of the Med8C/18/20 subcomplex. The expression profiles for these med8CA,

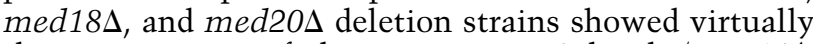
the same pattern of changes in mRNA levels (Fig. 4A). This is reflected in very high pairwise overall correlations (Fig. 4B).

For comparison, we repeated the analysis with two deletion strains that lacked genes for nonessential subunits Med2 or Med3 that reside in the tail module and also play a positive role in transcription (van de Peppel et al. 2005). Expression changes induced by Med2 or Med3 deletion correlate weakly with those induced by deletion of Med8C/18/20 components, although there is some overlap (Fig. 4A,B). Therefore, the Med8C/18/20 subcomplex regulates transcription of a specific subset of genes and forms a functional submodule in vivo. The similarity in expression profiles is in agreement with the idea that the only role of Med8C is to tether Med18/20 to the core head. These results highlight the possibility to correlate structural data with transcriptome profiles, thereby identifying proteins and protein domains that reside in the same functional module.

\section{Med8C/18/20 is required for transcription of specific genes}

Analysis of the expression profiles revealed that a total of 165 mRNA levels were significantly altered in all three deletion strains of the Med8C/18/20 submodule. Of those, 117 were down-regulated, and 48 were up-regulated, showing that the module predominantly acts as a positive factor in transcription, but can also act as a negative factor for certain genes. Of the down- and upregulated genes, $44 \%$ and $37 \%$, respectively, were not annotated in the gene ontology (GO) database (Fig. 4C; Ashburner et al. 2000). Since we had shown previously that Med8C/18/20 binds to the TATA-box-binding protein (TBP) in vitro (Lariviere et al. 2006), we were encouraged to unravel a molecular basis for Med8C/18/20 function by searching for reoccurring promoter elements or upstream motifs in the deregulated genes, and for common transcription factors known to regulate these genes. This search was, however, unsuccessful, suggesting a complex context-dependent mechanism of Med8C/ $18 / 20$ function.

We next analyzed the cellular function of genes regulated by Med8C/18/20 and by Med2 or Med3 with the GO Slim Mapper (Materials and Methods; Supplemental Table 2). Many of the affected biological processes, including amino acid derivative metabolism, carbohydrate metabolic process, and vitamin metabolic process, were overrepresented in both the Med8C/18/20-regulated and Med2/3-regulated genes (Fig. 4D; Supplemental Table 2), reflecting the partial overlap of the expression profiles (Fig. 4A). However, some biological processes were overrepresented only among Med8C/18/20- or Med2/ Med3-regulated genes. In particular, genes involved in conjugation were specifically Med8C/18/20-regulated, whereas genes involved in sporulation and cell wall organization and biogenesis were specifically Med2/Med3regulated (Fig. 4E). These findings are consistent with the previously reported involvement of Med18 in conjugation (Holstege et al. 1998) and with a large-scale functional genomics analysis of sporulation efficiency (Enyenihi and Saunders 2003).

\section{Down-regulation of nonactivated genes and basal promoter activity}

Down-regulation of conjugation genes in med8CA,

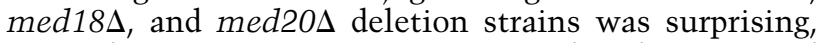
since their transcription was expected to be repressed under our experimental conditions. However, the same phenomenon was observed for other genes that should be repressed under optimal growth conditions. The GAL4 gene is further down-regulated in the med8CA, med18A, and med20s deletion strains, but not in the control strains lacking med2 or med3. Genes involved in the catabolism of serine and glycine are further down-regulated in all deletion strains, including CHA1, GCV1, GCV2, and GCV3. CHA1 shows the most down-regulated transcript levels, although it is expected to be expressed only under nitrogen-limiting conditions. Thus, Med8C/18/20 is required for low levels of transcription of nonactivated genes.

Taken together, many genes that are repressed under our growth conditions were apparently transcribed at a low level in a Med8C/18/20-dependent manner. The requirement of Med8C/18/20 for low-level transcription of nonactivated genes is consistent with the observation that even low levels of transcription require Mediator (Kornberg 2005; Takagi and Kornberg 2006). Although an indirect effect cannot be ruled out, our data suggest that Med8C/18/20 is important for basal promoter activity, and are consistent with a possible TBP interaction in vivo. A role of Mediator in basal transcription may be further investigated by similar analyses of other Media- 
A

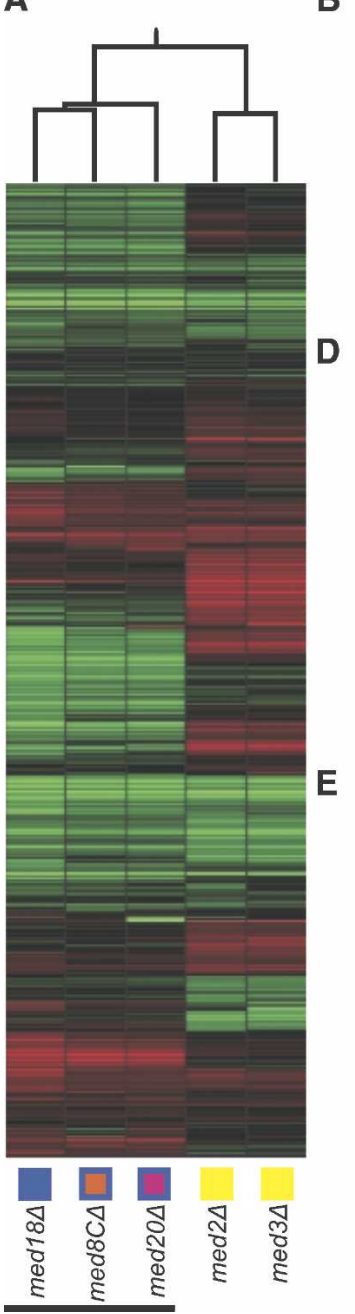

E
B

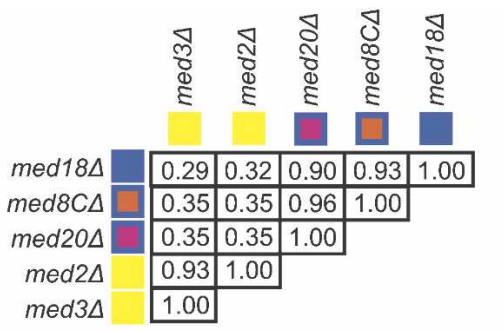

C

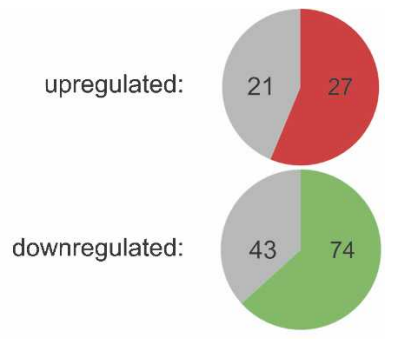

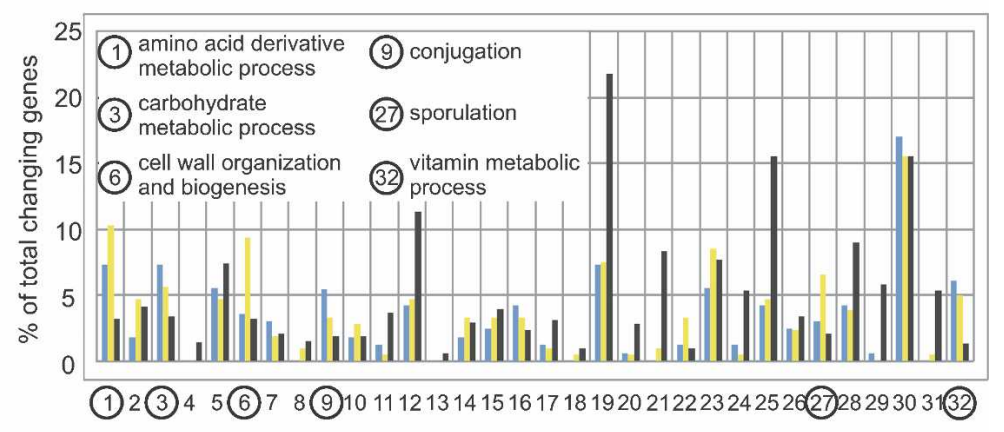

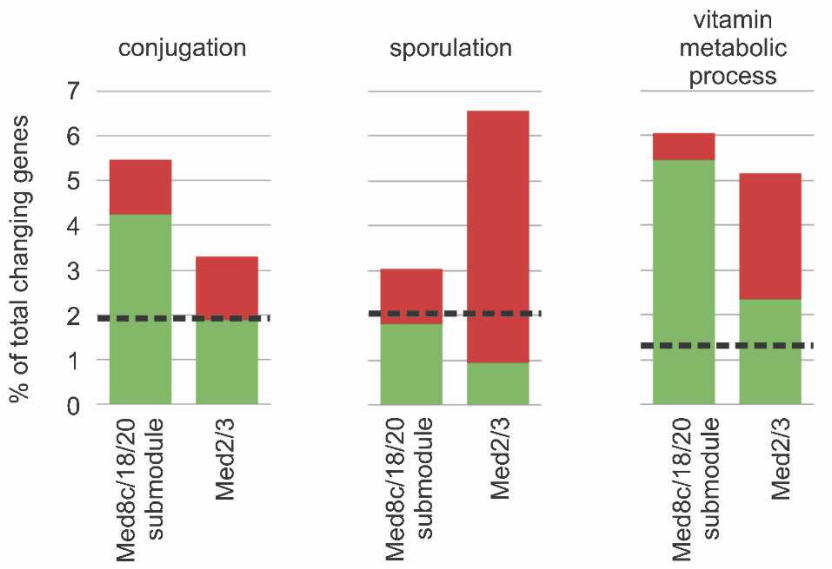

Figure 4. Transcriptome profiling analysis. $(A)$ Diagram of genes exhibiting significantly altered mRNA levels (vertical axis) for different Mediator deletion strains (horizontal axis), clustered alongside the med18s, med20s, and med8C $\Delta$ expression profiles (indicated by black bar). Changes in mRNA levels compared with the wild-type strain are depicted in red (up), green (down), or black (no change). (B) Pearson's

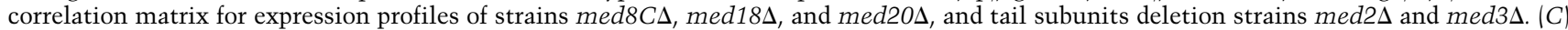
Number of significantly altered genes in all three deletion strains of the Med8C/18/20 submodule. Genes not annotated in the GO database are depicted in gray, up-regulated genes are indicated in red, and down-regulated genes are shown in green. $(D)$ Percentage of genes for which expression was significantly changed in all deletion mutants of the Med8C/18/20 (blue) submodule or Med2 and Med3 (yellow) compared with the percentage of the genome (black). All 32 biological processes from GO Slim Mapper are shown as follows: amino acid derivative metabolic process (1), anatomical structure morphogenesis (2), carbohydrate metabolic process (3), cell budding (4), cell cycle (5), cell wall organization and biogenesis (6), cellular homeostasis (7), cellular respiration (8), conjugation (9), cytokinesis (10), cytoskeleton organization and biogenesis (11), DNA metabolic process (12), electron transport (13), generation of precursor metabolites and energy (14), lipid metabolic process (15), meiosis (16), membrane organization and biogenesis (17), nuclear organization and biogenesis (18), organelle organization and biogenesis (19), protein catabolic process (20), protein modification process (21), pseudohyphal growth (22), response to stress (23), ribosome biogenesis and assembly (24), RNA metabolic process (25), signal transduction (26), sporulation (27), transcription (28), translation (29), transport (30), vesicle-mediated transport (31), and vitamin metabolic process (32). Overrepresented processes are marked with a circle. (E) Percentage of genes involved in conjugation, sporulation, and vitamin metabolic process for which expression was significantly changed in all deletion mutants of the Med8C/18/20 submodule or Med2 and Med3. Red and green histograms correspond to up- and down-regulated genes, respectively. The dotted line represents the percentage of the genome involved in the respective process.

tor submodules and transcriptome profiling of several mutant yeast strains under various growth conditions.

\section{Mediator modules contain submodules}

The Mediator head module is essential for cell viability, and a temperature-sensitive point mutation in the head leads to a global defect in transcription (Holstege et al. 1998). Here, we show that the head module contains the distinct conserved Med8C/18/20 submodule that is not essential for viability and regulates only a subset of genes. Identification and characterization of the Med8C/ $18 / 20$ submodule required a combination of structural biology, yeast genetics, biochemistry, and transcriptome analysis. Our results support the idea that the known Mediator modules head, middle, and tail contain distinct submodules with different functions that are involved in the regulation of different subsets of genes. 


\section{Structure-based system perturbation analysis of gene regulation}

More generally, we demonstrate how structural and functional information obtained on the molecular level in vitro can be correlated with changes on the systems level in vivo. In particular, the structure-guided design of mutant yeast strains enabled a precise disruption of molecular interactions and their functional analysis in vitro and in vivo. This approach is superior to the generally used gene deletion analysis, which does not take into account the consequences of such deletions for native protein complex structures. Most proteins reside in complexes (Gavin et al. 2002, 2006; Krogan et al. 2006), and gene deletion will often result in complex disintegration and malfunction, and thus in complicated changes of the transcriptome, which renders correlations between the molecular and the systems level difficult or impossible. In contrast, the structure-based systems perturbation analysis conducted here reduces the complexity of differential gene expression patterns, and facilitates the dissection of transcriptional coregulatory complexes into distinct functional submodules. In the future, this approach may be used for a reliable analysis of gene regulatory molecular networks. In particular, structure-based perturbation of cooperative molecular interactions could elucidate combinatorial and context-dependent gene regulatory mechanisms on the system level.

\section{Materials and methods}

TAP was performed essentially as described (Puig et al. 2001). Recombinant proteins were expressed in E. coli. For protein coexpression, multicistronic vectors were constructed as described (Lariviere et al. 2006). Proteins were purified by affinity chromatography, eventually followed by anion exchange chromatography and gel filtration for crystallization purposes. For Sc Med18/20, affinity purification was replaced by a precipitation with $30 \%$ saturated ammonium sulfate. Crystals of Sp Med18 and of the Med $8 \mathrm{C} / 18$ heterodimer were grown at $20^{\circ} \mathrm{C}$ in hanging drops over reservoirs containing $100 \mathrm{mM}$ Tris ( $\mathrm{pH} 8.5), 2 \mathrm{M}$ sodium acetate, and $2 \mathrm{M}$ sodium formate. The Med18 structure was solved by the singlewavelength anomalous dispersion method using selenomethionine-labeled protein crystals. The Med8C/Med18 structure was solved by molecular replacement using the Med18 structure as a model. For crystallographic details, see Supplemental Table 1. The Sp Med8C/18 structure was deposited in the Protein Data Bank under accession code 3C0T. Nuclear extracts were prepared from $3 \mathrm{~L}$ of culture as described (http:// www.fhcrc.org/labs/hahn). Plasmid transcription and analysis by primer extension were performed essentially as described (Ranish and Hahn 1991). Instead of the ${ }^{32} \mathrm{P}$-labeled lacI oligo, $0.125 \mathrm{pmol}$ fluorescently labeled 5 '-FAM-oligo was used. For gene expression profiling, all strains except med8CA were as described (van de Peppel et al. 2005). The med8CA strain is isogenic to the S288c strain. Truncation variant was prepared by introducing the adh1 terminator from pFA6a-13myckanMX6 (Longtine et al. 1998). Microarray data have been submitted to the ArrayExpress database (http://www.ebi.ac.uk/microarray) under accession number UMCU-MED-8. Detailed protocols can be found in the Supplemental Material.

\section{Acknowledgments}

We thank Larissa Wenzeck and Georg Arnold for help, and members of the Hahn laboratory for support. Part of this work was performed at the ESRF (Grenoble, France) and at the Swiss Light Source (SLS) at the Paul Scherrer Institute (Villigen, Switzerland). This work was supported by an EMBO long-term fellowship to L.L.; a Boehringer-Ingelheim Ph.D. fellowship to M.S.; by grants of the Deutsche Forschungsgemeinschaft, the Sonderforschungsbereich SFB646, and the Fonds der chemischen Industrie to P.C. and K.S.; by the EU grant 3D repertoire, contract number LSHG-CT-2005-512028 to P.C.; and by grant 5R01GM053451 to S.H.

\section{References}

Ashburner, M., Ball, C.A., Blake, J.A., Botstein, D., Butler, H., Cherry, J.M., Davis, A.P., Dolinski, K., Dwight, S.S., Eppig, J.T., et al. 2000. Gene ontology: Tool for the unification of biology. Nat. Genet. 25: $25-29$.

Backstrom, S., Elfving, N., Nilsson, R., Wingsle, G., and Bjorklund, S. 2007. Purification of a plant mediator from Arabidopsis thaliana identifies PFT1 as the Med25 subunit. Mol. Cell 26: 717-729.

Beve, J., Hu, G.Z., Myers, L.C., Balciunas, D., Werngren, O., Hultenby, K., Wibom, R., Ronne, H., and Gustafsson, C.M. 2005. The structural and functional role of Med5 in the yeast Mediator tail module. J. Biol. Chem. 280: 41366-41372.

Bjorklund, S. and Gustafsson, C.M. 2005. The yeast Mediator complex and its regulation. Trends Biochem. Sci. 30: 240-244.

Boube, M., Joulia, L., Cribbs, D.L., and Bourbon, H.-M. 2002. Evidence for a mediator of RNA polymerase II transcriptional regulation conserved from yeast to man. Cell 110: 143-151.

Dotson, M.R., Yuan, C.X., Roeder, R.G., Myers, L.C., Gustafsson, C.M., Jiang, Y.W., Li, Y., Kornberg, R.D., and Asturias, F.J. 2000. Structural organization of yeast and mammalian mediator complexes. Proc. Nat1. Acad. Sci. 97: 14307-14310.

Enyenihi, A.H. and Saunders, W.S. 2003. Large-scale functional genomic analysis of sporulation and meiosis in Saccharomyces cerevisiae. Genetics 163: 47-54.

Gavin, A.C., Bosche, M., Krause, R., Grandi, P., Marzioch, M., Bauer, A., Schultz, J., Rick, J.M., Michon, A.M., Cruciat, C.M., et al. 2002. Functional organization of the yeast proteome by systematic analysis of protein complexes. Nature 415: 141-147.

Gavin, A.C., Aloy, P., Grandi, P., Krause, R., Boesche, M., Marzioch, M., Rau, C., Jensen, L.J., Bastuck, S., Dumpelfeld, B., et al. 2006. Proteome survey reveals modularity of the yeast cell machinery. Nature 440: $631-636$

Guglielmi, B., van Berkum, N.L., Klapholz, B., Bijma, T., Boube, M., Boschiero, C., Bourbon, H.-M., Holstege, F.C.P., and Werner, M. 2004. A high resolution protein interaction map of the yeast Mediator complex. Nucleic Acids Res. 32: 5379-5391.

Holstege, F.C., Jennings, E.G., Wyrick, J.J., Lee, T.I., Hengartner, C.J., Green, M.R., Golub, T.R., Lander, E.S., and Young, R.A. 1998. Dissecting the regulatory circuitry of a eukaryotic genome. Cell 95: 717728.

Kang, J.S., Kim, S.H., Hwang, M.S., Han, S.J., Lee, Y.C., and Kim, Y.J. 2001. The structural and functional organization of the yeast mediator complex. J. Biol. Chem. 276: 42003-42010.

Koleske, A.J., Buratowski, S., Nonet, M., and Young, R.A. 1992. A novel transcription factor reveals a functional link between the RNA polymerase II CTD and TFIID. Cell 69: 883-894.

Kornberg, R.D. 2005. Mediator and the mechanism of transcriptional activation. Trends Biochem. Sci. 30: 235-239.

Krogan, N.J., Cagney, G., Yu, H., Zhong, G., Guo, X., Ignatchenko, A., Li, J., Pu, S., Datta, N., Tikuisis, A.P., et al. 2006. Global landscape of protein complexes in the yeast Saccharomyces cerevisiae. Nature 440: 637-643.

Lariviere, L., Geiger, S., Hoeppner, S., Rother, S., Strasser, K., and Cramer, P. 2006. Structure and TBP binding of the Mediator head subcomplex Med8-Med18-Med20. Nat. Struct. Mol. Biol. 13: 895901.

Lee, Y.C., Park, J.M., Min, S., Han, S.J., and Kim, Y.J. 1999. An activator binding module of yeast RNA polymerase II holoenzyme. Mol. Cell. Biol. 19: 2967-2976.

Longtine, M.S., McKenzie III, A., Demarini, D.J., Shah, N.G., Wach, A., Brachat, A., Philippsen, P., and Pringle, J.R. 1998. Additional modules for versatile and economical PCR-based gene deletion and modification in Saccharomyces cerevisiae. Yeast 14: 953-961.

Malik, S. and Roeder, R.G. 2000. Transcriptional regulation through Mediator-like coactivators in yeast and metazoan cells. Trends Biochem. Sci. 25: 277-283.

Naar, A.M., Lemon, B.D., and Tjian, R. 2001. Transcriptional coactivator complexes. Annu. Rev. Biochem. 70: 475-501.

Puig, O., Caspary, F., Rigaut, G., Rutz, B., Bouveret, E., Bragado-Nilsson, E., Wilm, M., and Seraphin, B. 2001. The tandem affinity purification (TAP) method: A general procedure of protein complex purification. Methods 24: 218-229. 
Ranish, J.A. and Hahn, S. 1991. The yeast general transcription factor TFIIA is composed of two polypeptide subunits. J. Biol. Chem. 266: 19320-19327.

Ranish, J.A., Yudkovsky, N., and Hahn, S. 1999. Intermediates in formation and activity of the RNA polymerase II preinitiation complex: Holoenzyme recruitment and a postrecruitment role for the TATA box and TFIIB. Genes \& Dev. 13: 49-63.

Singh, H., Erkine, A.M., Kremer, S.B., Duttweiler, H.M., Davis, D.A., Iqbal, J., Gross, R.R., and Gross, D.S. 2006. A functional module of yeast mediator that governs the dynamic range of heat-shock gene expression. Genetics 172: 2169-2184.

Takagi, Y. and Kornberg, R.D. 2006. Mediator as a general transcription factor. J. Biol. Chem. 281: 80-89.

Takagi, Y., Calero, G., Komori, H., Brown, J.A., Ehrensberger, A.H., Hudmon, A., Asturias, F., and Kornberg, R.D. 2006. Head module control of mediator interactions. Mol. Cell 23: 355-364.

Thompson, C.M., Koleske, A.J., Chao, D.M., and Young, R.A. 1993. A multisubunit complex associated with the RNA polymerase II CTD and TATA-binding protein in yeast. Cell 73: 1361-1375.

van de Peppel, J., Kettelarij, N., van Bakel, H., Kockelkorn, T.T.J.P., van Leenen, D., and Holstege, F.C.P. 2005. Mediator expression profiling epistasis reveals a signal transduction pathway with antagonistic submodules and highly specific downstream targets. Mol. Cell 19: 511-522. 


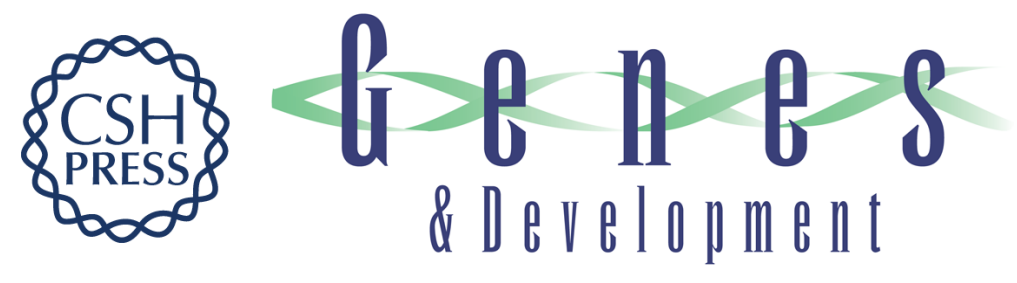

\section{Structure-system correlation identifies a gene regulatory Mediator submodule}

Laurent Larivière, Martin Seizl, Sake van Wageningen, et al.

Genes Dev. 2008, 22:

Access the most recent version at doi:10.1101/gad.465108

\section{Supplemental http://genesdev.cshlp.org/content/suppl/2008/03/12/22.7.872.DC1 Material}

References This article cites 28 articles, 9 of which can be accessed free at: http://genesdev.cshlp.org/content/22/7/872.full.html\#ref-list-1

\section{License}

Email Alerting

Receive free email alerts when new articles cite this article - sign up in the box at the top Service right corner of the article or click here.

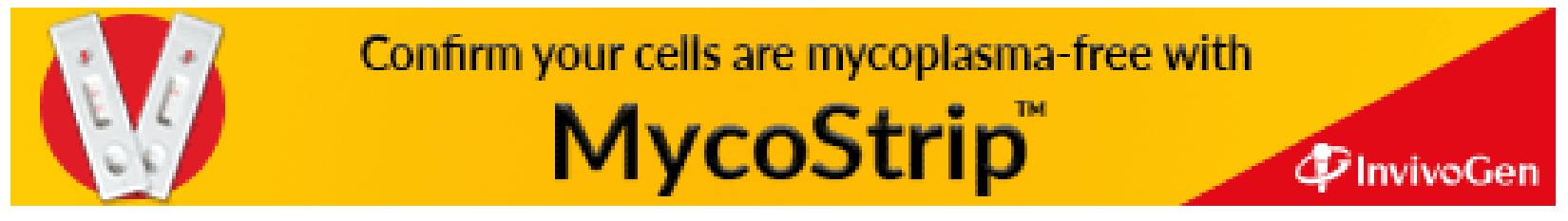

